\title{
Análisis del emprendimiento turístico en Ecuador y su impacto en la sociedad. Caso de estudio: empresas familiares en la parroquia de Mindo
}

\section{Touristic ventures analysis and it's society impact. Study cases: family ventures at Mindo's parish}

\author{
Daniela Polanco $^{1 *}$, María Belén Araujo ${ }^{1}$, Carolina Portugal ${ }^{1}$, Jaime Guamialamá ${ }^{1}$, Marcos Valdes $^{1}$, Diego Salazar Duque ${ }^{1}$
}

1 Universidad UTE. Rumipamba y Burgeios, Quito. 170508. Ecuador

Autor para correspondencia: daniela.polanco@ute.edu.ec

\begin{abstract}
Resumen
Mindo es una parroquia del cantón San Miguel de los Bancos, ubicada en el valle del Bosque Protector Mindo Nambillo; cuenta con gran diversidad de flora y fauna que la han posicionado como un destino turístico de naturaleza. Esta dinámica ha generado el desarrollo de emprendimientos turísticos, en su mayoría familiares, que aportan con la oferta del destino. Sin embargo, a pesar de ser un destino turístico importante para el país no ha podido despuntar con sus emprendimientos y mantiene una oferta comercial limitada. Por lo tanto, el objetivo de la presente investigación es analizar el emprendimiento turístico en Ecuador, enfocado en la parroquia de Mindo, y su impacto en la sociedad actual en la que se desenvuelve, examinando la dinámica de los emprendimientos turísticos familiares. Para ello se consideró una metodología con enfoque cualitativo y cuantitativo de carácter descriptivo. Se levantó información apegada a variables como: confianza individual, innovación, asunción de riesgo, toma de decisiones, creación de empresa, tipos de emprendimientos y relaciones entre clientes, proveedores y competidores que aportan con el análisis del mismo. Entre las principales conclusiones que se obtuvieron del estudio se encontró que la innovación es un factor fundamental y un elemento que no se percibe en la dinámica turística de la parroquia; que la falta de análisis técnicos de riesgos no aporta con información pertinente para una toma de decisiones acertada; $\mathrm{y}$, finalmente, que el trabajo conjunto entre los actores del turismo en la parroquia no apunta a objetivos conjuntos lo cual genera una falta de consensos y decisiones que permitan el desarrollo de un destino turístico competitivo.
\end{abstract}

Palabras clave: Mindo, emprendimientos familiares, dinámica turística, oferta turística, destino competitivo.

\begin{abstract}
Mindo is a parish located at San Miguel de los Bancos, next to the Preservation Forrest Mindo, Nambillo. It is positioned as a touristic destination, diverse in flora and fauna. This dynamism has generated the development of touristic ventures, most of them managed by families that contribute to the destination offer. Even though Mindo is considered an important destination in Ecuador, it does not generate enough income for a local sustainable development. The aim of this study is to analyze the tourism entrepreneurship of the parish of Mindo, as well as examine actual conditions in which they develop. Qualitative, quantitative and descriptive approach were used in the study, and the following variables were analyzed: individual trust, innovation, risk assumption, decision making, business creation, types of ventures and relations between clients, providers, and competitors who contributed with the study. The most relevant conclusions determine that innovation is a key factor that is not perceived at Mindo's touristic dynamics; the lack of technical risk analysis gives poor
\end{abstract}


information to make decisions. Finally, the parish and groups of interest are not working together with a defined horizon, this generates nonexistence of agreements and decisions that does not allow Mindo to become a competitive destination.

Keywords: Mindo, ventures, touristic dynamism, tourist offer, competitive destination

\section{Introducción}

En la actualidad una de las actividades clave en el desarrollo económico y social de una región es el emprendimiento, por su alto componente de innovación en sus productos y servicios, los cuales buscan satisfacer las necesidades y exceder las expectativas de los consumidores. Este, a su vez, reside en aspectos como la transformación de la voluntad humana en el acto, las decisiones del emprendedor sobre la base de productos y servicios necesarios, y las representaciones y mentalidades sobre la forma de concebir las oportunidades de innovación, creación y negocio (Rodríguez, 2009) que requiere el entorno.

Sobre esta postura, existen diferentes tipos de emprendimientos que tienen su propia dinámica de acuerdo con la actividad en la que cada uno se desenvuelve.

Para el caso de este estudio se toma en cuenta los emprendimientos turísticos, los cuales se forman por las condiciones externas e iniciativas propias en las que se incluye: la planificación, organización, dirección, control, y construcción de un negocio turístico (Fernández, 2009). En otras palabras, este tipo de emprendimiento se origina por una necesidad presente en los pobladores y con el objetivo de satisfacer las necesidades de turistas nacionales y extranjeros.

De acuerdo con Vázquez, Osorio y Arellano (2013), este tipo de emprendimiento está categorizado por su capacidad genérica para crear riqueza, desarrollo y bienestar; y a la actividad turística que se ofrezca en el mercado; entre estos se destacan:

- Emprendimiento pro-sostenible: Emprendimientos con una base socioeconómica y de organización más horizontal, existe una relación amistosa entre pequeñas empresas y su objetivo es la conservación del medio ambiente.

- Emprendimiento social: Son las empresas sociales que buscan la creación de emprendimientos innovadores y de satisfacer necesidades individuales.
- Emprendimiento ecológico: Son emprendimientos con objetivos exclusivos del cuidado de ecosistemas y biodiversidad.

Todos los emprendimientos tienen un eje común, puesto que no solamente buscan la generación de ingresos sino también un desarrollo social y cuidado ambiental de un territorio. En este sentido, muchos de los emprendimientos se han desarrollado endógenamente y con el fin de mejorar el ámbito social-familiar.

Si bien el emprendimiento se ha convertido en un potencial motor de la economía del país, se espera que el impacto en la sociedad que éste genere sea mayor. De acuerdo con el estudio realizado en el año 2009 por el Monitor Global de Emprendimiento (GEM, 2018), el índice de la actividad emprendedora temprana conocida como TEA, fue del 29,62\% en Ecuador (Global Entepreneurship Monitor). Es decir que los emprendimientos surgen en su mayoría por necesidad o por oportunidades temporales, sin embargo, no están catalogados como verdaderos negocios que puedan tener éxito y que sean sostenibles en el tiempo.

Bajo esta consideración, en la actualidad, la industria del turismo en Ecuador se encuentra en crecimiento, siendo Mindo uno de los destinos clave, tanto por su diversidad como por los servicios que se ofertan. En este sector se han instalado un importante número de emprendimientos turísticos que, lamentablemente, debido a diversos factores externos e internos del negocio, y que se exponen a lo largo de la presente investigación, no han podido ser sostenibles en el tiempo. En este sentido, el impacto de los emprendimientos en la sociedad debe ser positivo para que el destino pueda volverse atractivo. Los resultados que se presentan pretenden mejorar dichos servicios y en general el potencial de emprendimiento en la zona.

El emprendimiento forma parte del sistema turístico, el cual es la base fundamental de la planificación turística y gestión de un destino; dentro de éste existen subsistemas que funcionan aislada- 
mente y en conjunto para que toda la actividad y el destino puedan ser competitivos. Para su desarrollo es importante contar con atractivos relevantes, infraestructura, servicios y el apoyo y participación de la población. Gracias a este sistema se genera una cadena de servicios que aporta con generación de empleo y, consecuentemente, mejores ingresos (Boullón, 2006).

En este sentido, el Ecuador se destaca por ser un país que cuenta con un valioso potencial para realizar actividades turísticas por su riqueza natural y cultural. "En el año 2018 llegaron 2.248.538, y 1.472 .469 turistas, sin considerar el ingreso de venezolanos, lo que representó un crecimiento del 14\% con respecto al 2016" (Ministerio de Turismo, 2018). Uno de los destinos con mayor potencial turístico es la parroquia de Mindo ubicada al noroccidente de Quito, que cuenta con alrededor de 19200 hectáreas de bosques subtropicales. Además, posee recursos naturales importantes como cascadas y senderos que permiten el avistamiento de biodiversidad (Gobierno Autónomo Descentralizado de la Parroquia de Mindo, 2015). La actividad turística dentro de la parroquia es una de las principales actividades económicas la cual, además, fomenta en cierta medida el cuidado de los recursos naturales (Zalles, 2018).

Estas actividades turísticas están fortalecidas por la riqueza de avifauna del lugar, por tal motivo es una parroquia considerada como zona de importancia para la conservación de las aves (IBA), en América del Sur (Carpio \& Sánchez, 2013), debido a que se encuentra en dos bioregiones importantes: la del Chocó que viene desde la costa pacífica de Colombia y la de las estribaciones occidentales de los Andes -Ecuador y Colombia- (Gobierno Autónomo Descentralizado de la Parroquia de Mindo, 2015). En consecuencia, los servicios turísticos ofertados están encaminados a satisfacer una demanda exigente que busca servicios de calidad y que les permita pernoctar en un lugar con todas las facilidades y a su vez disfrutar de actividades de turismo de aventura y aviturismo.

El gobierno del Ecuador, en este sentido ha tratado de desarrollar políticas encaminadas a la generación de productos turísticos priorizados. En el año 2016 uno de los productos fue el aviturismo dentro del turismo de naturaleza, sin embargo, aunque se establecieron rutas en varios destinos con diversidad de avifauna no se consideró a Mindo en la planificación (Ministerio de Turismo, 2017). Para el 2017, el Ministerio de Turismo desarrolló una mesa de trabajo interinstitucional la cual estaba conformada por el Gobierno Autónomo Descentralizado (GAD) Municipal de San Miguel de Los Bancos, con las direcciones de Gestión Ambiental y Riesgos, Desarrollo Social y Económico, y la Comisaría Municipal; GAD Parroquial de Mindo; Policía Nacional; Tenencia Política de Mindo; GAD Provincial de Pichincha; Dirección Provincial de Pichincha del Ministerio del Ambiente. Esta mesa tenía como objetivo buscar soluciones a la problemática turística de Mindo y trabajar conjuntamente para mejorar la actividad. Para el año 2018, dentro de los productos turísticos priorizados no se encuentra el aviturismo como tal, pero sí la observación de flora y fauna. Además, Mindo está considerada como una de las parroquias para formar parte de los pueblos mágicos del Ecuador y se creó como producto una ruta de observación de aves a nivel país la cual incluye la parroquia. (Ministerio de Turismo, 2019b)

Este interés particular ha dado lugar al nacimiento de iniciativas que tratan de satisfacer una creciente demanda. De acuerdo con el Catastro Nacional de Actividades 2019 (Ministerio de Turismo, 2019a), existen actualmente 129 establecimientos turísticos entre agencias de viajes, operadoras, restaurantes y sitios de alojamiento. Sus propietarios en su mayoría son nacionales y un pequeño porcentaje inversores extranjeros. Estos negocios, acorde a la información del GAD de Mindo, en su mayoría son empíricos que se iniciaron entre familiares de la zona. El problema surge en conjunto con el crecimiento de la parroquia, los servicios y la infraestructura que ofrece, mismos que se han incrementado con el paso de los años. Sin embargo, es notoria la carencia de formación en el manejo de negocios turísticos (Gobierno Autónomo Descentralizado de la Parroquia de Mindo, 2015).

Por lo tanto, el objetivo de la investigación es analizar el emprendimiento turístico en Ecuador de la parroquia de Mindo y su impacto en la sociedad actual que aporte con información actualizada para el desarrollo y mejora del perfil del emprendedor turístico, a fin de que contribuya a la creación de emprendimientos y empresas dedicados a la prestación de servicios y a la mejora de la competitividad del destino. 


\section{Materiales y métodos}

Dado que el turismo es una actividad que se desarrolla sobre la base del sistema turístico, se integran variables, tales como: demanda, oferta ${ }^{1}$, infraestructura, superestructura, equipamiento, comunidad receptora, etc. Es indispensable definir estrategias, las cuales permiten que las organizaciones puedan tomar decisiones enfocadas en un mejoramiento continuo y que resulte en la creación de escenarios laborales que aporten con el desarrollo de las familias y de la parroquia.

De este modo, la sinergia que debe producirse entre los grupos de interés, así como los emprendedores turísticos, debe llevarse a cabo de la manera más objetiva posible para que el servicio final que se presenta al turista sea atractivo. Por lo tanto, se analiza la estructura de estas relaciones que es fundamental para que este hecho ocurra.

La investigación propuesta se realizó en campo, lo que permitió la comprensión real de las situaciones que se experimentan en la parroquia de Mindo. Para ello se considera un estudio con enfoque cuantitativo que levanta la información mediante un método descriptivo y se contrasta los resultados con un estudio cualitativo a través de entrevistas no estructuradas. Al tratarse del análisis de los emprendimientos familiares, su dinámica se centra en el estudio de aspectos sociales, locales y habituales.

Para el estudio cuantitativo se tomaron en cuenta 87 establecimientos turísticos, los cuales, de acuerdo con el GAD parroquial, son familiares. A estos se les aplicó una encuesta con cinco niveles de evaluación acorde a la escala de Likert. Las variables que fueron analizadas están basadas en el perfil del emprendedor investigado por los autores Silveira y Silveira, quienes hacen un estudio de la autoconfianza en los emprendedores y mencionan que su perfil está determinado por diferentes factores: innovación, la asunción de riesgos, la toma de decisiones, la creación de empresas y autoconfianza son factores positivos en el perfil del emprendedor (Silveira \& Silveira, 2017).

De esta manera, en el instrumento de la encuesta aplicada a los emprendedores de negocios turísticos, se desarrollaron 31 preguntas cerradas para de- terminar: confianza individual, innovación, asunción de riesgo, toma de decisiones, y la relación con el proveedor, el cliente y competidores. Estas fueron trianguladas con la información cualitativa resultado de la observación no participante y la aplicación de encuestas semiestructuradas a ocho emprendedores turísticos familiares de la parroquia.

\section{Resultados}

Una vez desarrolladas las encuestas y luego de haber conversado con los emprendedores se obtuvieron datos importantes sobre el emprendimiento turístico de la parroquia de Mindo y su impacto en la sociedad actual.

\section{Tipos de establecimientos}

De los 87 emprendimientos investigados se identifican: 26 hosterías $(30 \%)$; 20 restaurantes (23,33\%); 17 hoteles $(20 \%) ; 12$ cafeterías $(13,33 \%)$; 3 agencias de viajes $(3,33 \%)$; y otros 9 emprendimientos $(10 \%)$ relacionados con actividades turísticas. Un 33,33\% de estos establecimientos se encuentran funcionando entre dos y cinco años; un 43,33\% entre seis y diez años; $16,67 \%$ entre once y veinte años; y un 6,67\% más de 20 años.

\section{Confianza individual}

De acuerdo con la Tabla 1, el 70\% del total de encuestados señalan que están de acuerdo en emprender en la parroquia de Mindo. El 30\% restante se enfocan en los problemas relacionados con el apoyo por parte del gobierno local, problemas económicos y de experticia en el turismo. De igual forma, el $66 \%$ del total de los encuestados están de acuerdo en superar las adversidades que se presentan, las cuales están enmarcadas en aspectos económicos o mejoramiento de infraestructura. El 34\% no tiene la capacidad de superar las adversidades debido a la falta de conocimiento de turismo. Por otro lado, el $83 \%$ del total de encuestados están de acuerdo en enfrentar los retos que exige el turismo en la parroquia de Mindo, considerando las expectativas del turista que visita la zona, la seguridad que el establecimiento proyecta en el momento de la prestación del servicio. Finalmente, el $83 \%$ del total de encuestados están de acuerdo en realizar 
un proceso de toma de decisiones conjuntas para el bienestar de la parroquia.

Según los entrevistados, todos han tenido la visión de que sus emprendimientos crezcan, no obstante, han tenido que pasar por un periodo mínimo de 5 años para empezar a tener estabilidad (Entrevista H.L., 2018). La mayor parte tuvo problemas al inicio por la falta de conocimiento en la parte legal para la constitución de su empresa y porque la inversión era bastante alta (Entrevista S.L., 2018).

Tabla 1. Confianza individual

\begin{tabular}{|l|c|c|c|c|c|}
\hline Criterio & $\begin{array}{c}\text { Totalmente } \\
\text { desacuerdo }\end{array}$ & $\begin{array}{c}\text { Un poco des- } \\
\text { acuerdo }\end{array}$ & $\begin{array}{c}\text { Ni de acuerdo ni } \\
\text { desacuerdo }\end{array}$ & De acuerdo & $\begin{array}{c}\text { Totalmente de } \\
\text { acuerdo }\end{array}$ \\
\hline Oportunidades & $0 \%$ & $13 \%$ & $17 \%$ & $30 \%$ & $40 \%$ \\
\hline Adversidades & $7 \%$ & $7 \%$ & $20 \%$ & $30 \%$ & $36 \%$ \\
\hline Acepto de retos & $1 \%$ & $3 \%$ & $13 \%$ & $23 \%$ & $60 \%$ \\
\hline $\begin{array}{l}\text { Posición de un tema para } \\
\text { la toma de decisiones }\end{array}$ & $1 \%$ & $6 \%$ & $10 \%$ & $20 \%$ & $63 \%$ \\
\hline
\end{tabular}

\section{Innovación}

En cuanto a la innovación, la Tabla 2 muestra los siguientes resultados: el $80 \%$ del total de encuestados están de acuerdo en innovar la prestación de servicios turísticos en la parroquia de Mindo. De acuerdo con los entrevistados, cada emprendimiento tiene diferentes herramientas de innovación; la mayoría se enfocan en la publicidad de la parroquia o promoción de sus negocios y no en mecanismos de innovación. En pocos casos consideran que es importante investigar y recorrer otros negocios turísticos a nivel nacional o internacional, como ejemplo para Mindo (Entrevista, T.O y J.P., 2018), lo cual se hace difícil debido a la situación económica, por tanto, sus procesos de innovación no se han dado de acuerdo con las nuevas tendencias del turismo.

De igual forma, el intercambio de ideas mejora la relación para incrementar la capacidad laboral. En este sentido, el $83 \%$ está de acuerdo en que crear, mantener y mejorar una relación laboral positiva entre todos los emprendedores turísticos de la zona mejora sin duda la capacidad laboral diaria. Además, consideran que una estrategia de innovación es generar trabajo local "Para nosotros lo mejor es tener personal local, el $100 \%$ de nuestro negocio es con personas de Mindo y eso le da un plus a nuestro servicio" (Entrevista, C.A., 2018). El $67 \%$ de la totalidad de encuestados bus- can soluciones fáciles y prácticas que resuelvan sus problemas. En tanto que el 33\% restante, por lo general tratan de buscar ayuda dentro de la parroquia o a emprendedores que brinden el mismo servicio. Además, esta búsqueda de soluciones se da en primera instancia dentro de cada familia debido a la dinámica del emprendimiento.

En este punto es necesario considerar que el emprendedor de la parroquia de Mindo reflexiona que existen buenas oportunidades de negocio en el sector. El 80\% está de acuerdo en que el sector se presta para implantar negocios turísticos, y que es prioritario contar con una organización efectiva del mismo para que genere impacto en la parroquia. Los emprendedores que no están atraídos por nuevas oportunidades manifestaron que se debe a la existencia de una amplia oferta dentro de la parroquia lo cual conlleva que cada vez tengan menos turistas.

Finalmente, el $77 \%$ de los emprendimientos encuestados se sienten motivados al momento de trabajar con profesionales innovadores, esto denota la importancia para ellos de contar con el apoyo profesional oportuno. Para los encuestados, en este sentido, la ayuda del Ministerio de Turismo es indispensable, "sobre todo en temas de atención al cliente y nuevas tendencias del turismo para poner en práctica en los emprendimientos" (Entrevista, O.T., 2018). 
Tabla 2. Innovación

\begin{tabular}{|l|c|c|c|c|c|}
\hline Criterio & $\begin{array}{c}\text { Totalmente } \\
\text { desacuerdo }\end{array}$ & $\begin{array}{c}\text { Un poco } \\
\text { desacuerdo }\end{array}$ & $\begin{array}{c}\text { Ni de acuerdo } \\
\text { ni desacuerdo }\end{array}$ & De acuerdo & Totalmente de acuerdo \\
\hline Innovar la profesión & $3 \%$ & $10 \%$ & $7 \%$ & $33 \%$ & $47 \%$ \\
\hline Fomento de intercambio de ideas & $0 \%$ & $10 \%$ & $7 \%$ & $20 \%$ & $63 \%$ \\
\hline $\begin{array}{l}\text { Búsqueda de soluciones a los } \\
\text { problemas }\end{array}$ & $0 \%$ & $13 \%$ & $20 \%$ & $30 \%$ & $37 \%$ \\
\hline $\begin{array}{l}\text { Atracción de nuevas oportunidades } \\
\text { en el negocio }\end{array}$ & $0 \%$ & $7 \%$ & $13 \%$ & $37 \%$ & $43 \%$ \\
\hline $\begin{array}{l}\text { Trabajo con profesionales } \\
\text { innovadores }\end{array}$ & $0 \%$ & $13 \%$ & $10 \%$ & $27 \%$ & $50 \%$ \\
\hline
\end{tabular}

\section{Asunción de riesgo}

Con relación a la asunción de riesgo, sus resultados se presentan en la Tabla 3. El 60\% tiene un análisis intuitivo mayor a los planes estratégicos o de negocios, en tanto que el $40 \%$ prefiere los planes técnicos de expertos. Lo cual en muchos casos ha incidido para que los emprendimientos surjan. Estos planes por lo general vienen de la academia con tesis de grado y en menor medida de la contratación de personal calificado en el área. De los entrevistados, el riesgo al iniciar el negocio fue alto, considerando que en ningún caso se desarrolló un plan de negocios y primó el empirismo o miembros de la familia que cursaban la carrera de Turismo "Nosotros empezamos de cero y sin saber mucho del tema, con el tiempo hemos ido perfeccionando y mejorando" (Entrevista, S.P., 2018).

En cuanto al proceso de gestión y reducción de riesgos, el $47 \%$ identifica que es importante va- lorarlo en un emprendimiento. Sin embargo, para los emprendedores de Mindo esto ha sido un trabajo de tenacidad en el tiempo, debido a la dinámica de la actividad turística que se desarrolla. Los deportes de aventura tienen intrínsecamente un riesgo el cual se ha logrado controlar con el fin de que éstos sean de calidad y seguridad para el turista. Por último, el 46\% coinciden en que el negocio se define por factores ocasionales, enlazados principalmente a los concursos del conteo de aves y a las actividades de turismo de aventura que entraron en boga en los últimos años. Esto permitió que muchos emprendimientos surjan en poco tiempo. Sin embargo, al ser un factor ocasional se les ha dificultado mantenerse en el tiempo. No obstante, el 34\% que está parcialmente de acuerdo, son emprendedores que han aprovechado las oportunidades ocasionales y que a su vez han podido desarrollar una planificación organizada y con visión a largo plazo del negocio.

Tabla 3. Asunción de riesgo

\begin{tabular}{|l|c|c|c|c|c|}
\hline Criterio & $\begin{array}{c}\text { Totalmente } \\
\text { desacuerdo }\end{array}$ & $\begin{array}{c}\text { Un poco } \\
\text { desacuerdo }\end{array}$ & $\begin{array}{c}\text { Ni de acuerdo } \\
\text { ni desacuerdo }\end{array}$ & De acuerdo & Totalmente de acuerdo \\
\hline Análisis intuitivo & $3 \%$ & $30 \%$ & $20 \%$ & $17 \%$ & $30 \%$ \\
\hline $\begin{array}{l}\text { Proceso de gestión y reducción } \\
\text { de riesgos }\end{array}$ & $17 \%$ & $10 \%$ & $13 \%$ & $33 \%$ & $27 \%$ \\
\hline $\begin{array}{l}\text { Factores de medición del } \\
\text { negocio }\end{array}$ & $18 \%$ & $3 \%$ & $33 \%$ & $23 \%$ & $23 \%$ \\
\hline
\end{tabular}

Toma de decisiones

En cuanto a la toma de decisiones, según resultados obtenidos y presentados en la Tabla 4, el
$60 \%$ de los encuestados está de acuerdo con la toma de decisiones creativas para sus emprendimientos en lugar de seguir parámetros establecidos. Esto se debe a la dinámica de la actividad turística la cual 
va evolucionando con el pasar del tiempo. Por otro lado, el $70 \%$ de los emprendedores considera que un cambio en el entorno creará una nueva oportunidad para el negocio, esta situación se ha dado en varias ocasiones y la vocación de los negocios se da conforme a las nuevas tendencias del mercado en la parroquia. No obstante, un 53\% de los empresarios afirman su preferencia por dar importancia a las decisiones relativas que tenga un tema fundamental para la toma de decisiones relacionadas con el turismo. Por último, el $87 \%$ de los consultados afirma que toma decisiones oportunas para el desarrollo de estrategias según las oportunidades que se presentan en el mercado.
Al ser emprendimientos familiares, la toma de decisiones está definida principalmente por los miembros de la familia fundadores del negocio. En su mayoría han sido hermanos. Por lo general empezaron entre tres a cinco personas y con el pasar del tiempo van disminuyendo. "Inicialmente 5, anteriormente las decisiones eran tomadas por los 5 integrantes de la familia, actualmente solo yo" (Entrevista, J.P., 2018).

La familia tiene un peso importante a la hora de hacer cualquier cambio en el negocio y por este motivo también se torna más complicado el desarrollo del mismo.

Tabla 4. Toma de decisiones

\begin{tabular}{|l|c|c|c|c|c|}
\hline \multicolumn{1}{|c|}{ Criterio } & $\begin{array}{c}\text { Totalmente } \\
\text { desacuerdo }\end{array}$ & $\begin{array}{c}\text { Un poco } \\
\text { desacuerdo }\end{array}$ & $\begin{array}{c}\text { Ni de acuerdo ni } \\
\text { desacuerdo }\end{array}$ & De acuerdo & $\begin{array}{c}\text { Totalmente de } \\
\text { acuerdo }\end{array}$ \\
\hline Soluciones creativas & $0 \%$ & $10 \%$ & $30 \%$ & $23 \%$ & $37 \%$ \\
\hline Cambio en el entorno & $4 \%$ & $13 \%$ & $13 \%$ & $30 \%$ & $40 \%$ \\
\hline Priorización de decisiones & $7 \%$ & $23 \%$ & $17 \%$ & $23 \%$ & $30 \%$ \\
\hline Desarrollo de estrategias & $0 \%$ & $0 \%$ & $13 \%$ & $20 \%$ & $67 \%$ \\
\hline
\end{tabular}

\section{Creación de empresa}

En la Tabla 5, el 73\% de los encuestados consideran que la selección de los miembros del negocio es lo más importante, aunque en los emprendimientos familiares por lo general pasan de generación en generación, están conscientes que se debe contar con elementos capacitados y con experiencia para que estos puedan funcionar. Los entrevistados consideran que las capacitaciones permanentes y el tener una buena contabilidad ha sido una fortaleza, y por esto algunos se encuentran creando empresas o ya las tienen, incluso tienen la idea de crecer o ponerse otro negocio.

De igual forma un $70 \%$ de los encuestados manifestó el interés por utilizar y aplicar sistemas y métodos de vigilancia para mejorar la producción y operación de la empresa con el fin de llevar un control de los costos de producción y operaciones en general. Por otro lado, el $73 \%$ de los encuestados están seguros que el tiempo es importante para planificar el futuro de su empresa. Sin embargo, en su mayoría no han tenido una planificación previa y se han aprovechado oportunidades definidas. La gestión se ha ido efectuando sobre la marcha, lo que ha causado también problemas difíciles de superar. Finalmente, el 66\% de los encuestados considera que el éxito de su negocio se da por la toma de decisiones rápidas, en tanto que el $37 \%$ no lo cree de esa manera, puesto que prefieren tomarse el tiempo y analizar las alternativas antes de tomar cualquier decisión.

Tabla 5. Creación de empresa

\begin{tabular}{|l|c|c|c|c|c|}
\hline \multicolumn{1}{|c|}{ Criterio } & $\begin{array}{c}\text { Totalmente } \\
\text { desacuerdo }\end{array}$ & $\begin{array}{c}\text { Un poco } \\
\text { desacuerdo }\end{array}$ & $\begin{array}{c}\text { Ni de acuerdo } \\
\text { ni desacuerdo }\end{array}$ & $\begin{array}{c}\text { De } \\
\text { acuerdo }\end{array}$ & $\begin{array}{c}\text { Totalmente de } \\
\text { acuerdo }\end{array}$ \\
\hline $\begin{array}{l}\text { Criterio de selección en el } \\
\text { negocio }\end{array}$ & $7 \%$ & $0 \%$ & $20 \%$ & $13 \%$ & $60 \%$ \\
\hline $\begin{array}{l}\text { Utilización de sistemas y } \\
\text { métodos de control }\end{array}$ & $3 \%$ & $7 \%$ & $20 \%$ & $7 \%$ & $63 \%$ \\
\hline Tiempo de planeación & $3 \%$ & $7 \%$ & $17 \%$ & $13 \%$ & $60 \%$ \\
\hline Toma de decisiones rápidas & $11 \%$ & $13 \%$ & $30 \%$ & $13 \%$ & $33 \%$ \\
\hline
\end{tabular}




\section{Relación con el proveedor, el cliente y competidores}

En cuanto a las diversas relaciones del negocio, la información presente en la Tabla 6 muestra que el $70 \%$ de los emprendedores está consciente de que deben tener una buena relación con los proveedores para que funcione un negocio. De igual forma el $76 \%$ de los empresarios manifiesta que la relación con los clientes es muy importante para el éxito empresarial. Finalmente, el $60 \%$ de los encuestados considera que los competidores deberían tener una buena relación y apoyarse en acciones conjuntas a diferencia de un $40 \%$ que no lo considera de esa manera y prefieren trabajar aisladamente.

Sobre la base de lo manifestado en las entrevistas, se afirma que en la parroquia las relaciones conjuntas entre el sector hotelero no están diseñadas en conjunto. Además, carecen de iniciativas conjun- tas con otro tipo de empresas turísticas, lo que se ha convertido en un impedimento para seguir creciendo como empresa.

Las estrategias aisladas hacen que la oferta de producto no se diversifique y que no exista un apoyo para el sector hotelero. En cuanto a este último criterio, el 54\% de los emprendedores comparte información sobre mercado, clientes y proveedores con otras empresas del sector hotelero. Esto lo hacen siempre y cuando exista una retroalimentación entre empresas, las estadísticas, así como la información que poseen los emprendimientos más grandes no es fácil obtenerla y se trabaja aisladamente. La situación es similar con la información de costos y nuevas técnicas de producción, puesto que cada empresa está enfocada en su desarrollo económico y en menor medida en un trabajo compartido que beneficie al destino Mindo.

Tabla 6. Relaciones

\begin{tabular}{|l|c|c|c|c|c|}
\hline Criterio & $\begin{array}{c}\text { Totalmente } \\
\text { desacuerdo }\end{array}$ & $\begin{array}{c}\text { Un poco } \\
\text { desacuerdo }\end{array}$ & $\begin{array}{c}\text { Ni de acuerdo ni } \\
\text { desacuerdo }\end{array}$ & De acuerdo & $\begin{array}{c}\text { Totalmente de } \\
\text { acuerdo }\end{array}$ \\
\hline $\begin{array}{l}\text { Buena relación con } \\
\text { proveedores }\end{array}$ & $3 \%$ & $7 \%$ & $20 \%$ & $20 \%$ & $50 \%$ \\
\hline $\begin{array}{l}\text { Buena relación con los } \\
\text { clientes }\end{array}$ & $4 \%$ & $7 \%$ & $13 \%$ & $13 \%$ & $63 \%$ \\
\hline $\begin{array}{l}\text { Buena relación con los } \\
\text { competidores }\end{array}$ & $0 \%$ & $20 \%$ & $20 \%$ & $20 \%$ & $40 \%$ \\
\hline $\begin{array}{l}\text { Información compartida con } \\
\text { competidores }\end{array}$ & $10 \%$ & $13 \%$ & $23 \%$ & $24 \%$ & $30 \%$ \\
\hline
\end{tabular}

\section{Discusión}

Mindo es un destino turístico que se ha desarrollado sobre la base de las actividades de aventura, la mayor parte de emprendimientos son hosterías que ofrecen diferentes tipos de servicios y comodidades para los turistas nacionales y extranjeros.

Los emprendedores están conscientes de las falencias que existen y de las estrategias que deberían gestionarse, no obstante, las acciones han sido aisladas y no existe un trabajo conjunto entre quienes conforman el sector. Esta falta de organización también genera el bajo apoyo por parte del gobierno local, para la mayoría de emprendimientos familiares su trabajo por varios años ha sido individual y no han tenido mayor aporte o incentivos por parte del sector público. Este apoyo se ha enfocado en capacitaciones por par- te del Ministerio de Turismo o visitas esporádicas de los técnicos (Entrevista, T.O., 2018). Aunque el 37\% menciona que los problemas han sido superados y el $60 \%$ asegura que la toma de decisiones es clave para el éxito de un emprendimiento.

El 80\% de los encuestados están conscientes de la importancia de la innovación, sin embargo, pocos conocen mecanismos de innovación, sus herramientas están basadas en el marketing, y se reconoce que los negocios existentes en Mindo son muy similares lo cual limita la oferta de servicios.

El $43 \%$ de la muestra se siente entusiasmado por incluirse en nuevos negocios, sin embargo, el $63 \%$ de los encuestados tienden a limitarse cuando surgen inconvenientes en el negocio, lo que reduce la capacidad de resiliencia del emprendedor. 
Por otra parte, para los emprendedores el análisis intuitivo es de suma importancia a la hora de tomar decisiones, sin embargo, es importante tener un equilibrio con el análisis de negocios que en la mayoría de emprendimientos no se ha realizado. Por esta razón, en Mindo no están dispuestos a correr riesgos.

Las relaciones entre los emprendimientos son de suma importancia para que se pueda beneficiar el destino, sin embargo, todavía no se ha roto la barrera entre los competidores y en su mayoría no trabajan en conjunto. Esto es un limitante para llevar a cabo gestiones turísticas dentro de la parroquia.

Los emprendedores turísticos familiares de Mindo, tienen altas expectativas para la generación de negocios, todos están conscientes de que viven en una zona rica en biodiversidad y que esto se presenta como una gran oportunidad. Sin embargo, factores externos como la falta de inversión, conocimiento en el área y principalmente el miedo al fracaso hacen que los procesos se caigan.

De los emprendimientos encuestados los que llegaron a ser empresas son los que han tenido posibilidades de créditos grandes, tienen algún familiar que estudió turismo o la posibilidad de aliarse con un inversionista externo. Por otra parte, los emprendimientos pequeños en muchos casos llevan más de diez años teniendo el mismo giro de negocio y no piensan en crecer o innovar.

\section{Conclusiones}

Los emprendimientos turísticos no son fáciles de desarrollar debido a la dinámica del sector y las

\section{Referencias}

Boullón, R. (2006). Planificación del espacio turístico. México: Editorial Trillas.

Carpio, D., \& Sánchez, K. (2013). Plan de mejoramiento de los servicios turísticos de la parroquia de Mindo ubicada en el cantón San Miguel de los Bancos, provincia de Pichincha. Quito: Escuela Politécnica del Ejército.

Fernández, J. (2009). Emprendedores turísticos locales y su intermediación en la esfera delas políticas necesidades cambiantes del mercado. En Mindo el turismo ha crecido debido a su riqueza natural, los pobladores están conscientes de su atractivo y por ende buscan mejorar la competitividad del destino.

La innovación es un factor clave para generar un destino turístico posicionado, no obstante, en Mindo no se percibe mucha innovación en los negocios familiares puesto que no poseen el personal capacitado y no destinan parte de sus ganancias a este objetivo concreto.

Se debería realizar un análisis de riesgos dentro de los emprendimientos con el fin de identificar a tiempo negocios que estén en proceso de cierre para evitar pérdidas a largo plazo.

Se ha identificado un perfil de emprendedor motivado y consciente de las acciones a llevarse a cabo con el fin de tener éxito, sin embargo, en la práctica el factor de riesgo es un limitante y hace que los negocios se estanquen.

Esta investigación ha marcado la necesidad de contar con un programa de educación permanente y actualización de conocimientos en hospitalidad y servicios a la población de Mindo que cuenta con emprendimientos turísticos en el sector. De esta manera será posible que se mejoren de manera continua los servicios que se ofertan en el sector, se realice un cronograma de capacitaciones en temas de común interés para los emprendedores que buscan innovar su oferta turística para el visitante de la zona y contribuir con el desarrollo económico del Ecuador.

públicas: el caso de Mallorca. Una aproximación desde lasociología histórica y política. PASOS Revista de Turismo y Patrimonio Cultural, 7(3), 37138. https://doi.org/10.25145/j.pasos.2009.07.026

Global Entepreneurship monitor (2018). Emprendimiento en el mundo. Obtenido de: https://gem. ufm.edu/emprendimiento-en-el-mundo/

Gobierno Autónomo Descentralizado de la Parroquia de Mindo. (2015). Plan de Ordenamiento 
Territorial. Mindo: Gobierno Autónomo Descentralizado de la Parroquia de Mindo.

Ministerio de Turismo. (2017). Informe Narrativo Rendición de Cuentas 2016. Quito: Dirección de Seguimiento de Planes, Programas y Proyectos. Ministerio de Turismo.

Ministerio de Turismo. (2019a). Consolidado Nacional de Actividades Turísticas. Quito: Ministerio de Turismo.

Ministerio de Turismo. (2019b). Rendición de cuentas 2018. Quito: Ministerio de Turismo.

Ministerio de Turismo. (2018). Reporte de llegadas de turistas. Quito.

Rodríguez, A. (2009). Nuevas perspectivas para entender el emprendimiento empresarial. Revista cientifica Pensamiento y Gestión, 26, 95-119.

Silveira, Y., \& Silveira, R. (2017). Antecedentes del perfil del emprendedor brasileño de Pymes de los clusters industriales. Estudio del factor autoconfianza. Revista Portuguesa e Brasileira de Gestão, 16(1), 22- 43.

Vázquez, D., Osorio, M., \& Arellano, A. (2013). Turismo desde el pensamiento sistémico. Investigaciones turísticas, 5, 1-28. https://doi. org/110.14198/INTURI2013.5.01

Zalles, J. (2018). Turismo basado en naturaleza y conservación biológica: decisiones de uso de suelo en Mindo. Letras Verdes, (23), 178-198. https:// doi.org/10.17141/letrasverdes.23.2018.2861

\section{Entrevistas}

G.P. Propietario establecimiento turístico

S.L. Propietario establecimiento turístico

S.P. Gerente establecimiento turístico.

H.L. Socio de establecimiento turístico

T.O. Propietaria de establecimiento turístico.

C.A. Propietario de establecimiento turístico.

J.P. Propietaria operadora turística.

O.T. Propietario operadora turística. 